\title{
Pulse propagation in a linear and nonlinear diatomic periodic chain: effects of acoustic frequency band-gap
}

Received: 9 December 2008 / Revised: 6 February 2009 / Published online: 21 April 2009

(C) The Author(s) 2009. This article is published with open access at Springerlink.com

\begin{abstract}
One-dimensional nonlinear crystals have been assembled from periodic diatomic chains of stainless steel cylinders alternated with Polytetrafluoroethylene spheres. This system allows dramatic changes of behavior (from linear to strongly nonlinear) by the application of compressive forces practically without changes to the geometry of the system. The relevance of classical acoustic band-gap, characteristic for a chain with linear interaction forces and derived from the dispersion relation of the linearized system, on the transformation of single and multiple pulses in linear, nonlinear and strongly nonlinear regimes is investigated with numerical calculations and experiments. The limiting frequencies of the acoustic band-gap for the investigated system with a constant precompression force are within the audible frequency range $(20-20,000 \mathrm{~Hz})$ and can be tuned by varying the particle's material properties, mass and initial compression. In the linear elastic chain the presence of the acoustic band-gap was apparent through a fast transformation of incoming pulses within very short distances from the end of the chain. It is interesting that pulses with relatively large amplitude (nonlinear elastic chain) exhibit qualitatively similar behavior indicating the relevance of the acoustic band gap also for the transformation of nonlinear signals. The effects of an in situ band-gap created by a mean dynamic compression are observed in the strongly nonlinear wave regime.
\end{abstract}

\section{Introduction}

The study of pulse propagation in a one-dimensional diatomic chain as well as multilayered and periodic samples with a linear interaction law between masses has been a topic of growing interest. This is because the frequency band-gaps affect the behavior of the system by prohibiting the propagation of acoustic waves in this part of the frequency spectrum [1-14]. Previous theoretical investigations have focused on frequency band-gaps [1-5], localized modes [6,7] or discrete gap breathers [8,9] in discrete linear and weakly nonlinear dynamic systems with various interaction laws between particles.

Investigations of band-gaps in discrete metamaterials vary widely in experimental applications. For example, band-gaps and strongly localized modes are present in transversely loaded strings with masses [6,10]

\author{
E. B. Herbold · V. F. Nesterenko \\ Department of Mechanical and Aerospace Engineering, \\ University of California at San Diego, La Jolla, CA 92093-0418, USA \\ J. Kim $\cdot$ V. F. Nesterenko $(\bowtie) \cdot S$. Y. Wang \\ Materials Science and Engineering Program, \\ University of California at San Diego, La Jolla, CA 92093-0418, USA \\ E-mail: vnesterenko@ucsd.edu \\ C. Daraio \\ Graduate Aeronautical Laboratories (GALCIT), Department of Applied Physics, \\ California Institute of Technology, Pasadena, CA 91125, USA
}


and diatomic chains composed of welded spheres (with band-gaps in the frequency range of 55-75 kHz) [12]. Layered structures composed from two disparate materials exhibit acoustic band-gaps and pass-bands that may be engineered to be very narrow or broad by introducing multiple periods into the layered structure [13]. Passbands have also been utilized in non-mechanical systems using a slow-wave microelectromechanical delay line in a chain of coupled resonators [14]. This capacitively coupled MEMS delay line has a band-pass response and signal group velocity as low as $10 \mathrm{~m} / \mathrm{s}$, which can be used as a filter in wireless RF or communication systems.

One-dimensional chains of elements interacting according to Hertz's contact law are appropriate models to investigate linear, weakly nonlinear and strongly nonlinear wave dynamics of chains with different arrangements of particles and under different conditions of dynamic loading and initial prestress [15-39]. A more general interaction law between particles was introduced in [40-42]. It should be mentioned that the existence of strongly nonlinear solitary waves was proven in the long-wave approximation [17,31] and for discrete chains [20] for more general strongly nonlinear interactions between particles than Hertz's law. The concept of a "sonic vacuum" was first introduced in [17] to emphasize the uniqueness of the types of strongly nonlinear materials which do not support classical sound waves without initial prestress.

The versatility of chains with nonlinear interaction forces derives from the "tunability" of the wave propagation regime, from linear to strongly nonlinear through an initial static compression. This a priori adjustability of acoustic band-gaps in strongly nonlinear diatomic systems $[43,44]$ allows for the development of various practical applications, ranging from acoustic filters, noise mitigation and absorption layers to vibration insulation and the phonon wave-guides.

Strongly nonlinear solitary waves (the main mode of signal propagation in weakly compressed chains) exhibit qualitatively different features than weakly nonlinear systems. They have a finite width [15-19,38] that is independent of the solitary wave amplitude, and a pulse speed that is much smaller than the bulk sound speed of the spheres composing the chains [31]. The speed of strongly nonlinear solitary waves can be below the range of sound speed of fluids and gases, as it was demonstrated for PTFE and stainless steel based granular systems [27,31,33-35,37]. The strongly nonlinear wave equations derived for uniform and heterogeneous diatomic chains $[15,31,43,44]$ represent the most general description of wave dynamics including the weakly nonlinear and linear cases.

It should be mentioned that self-demodulation of nonlinear pulses occur in chains of identical spherical particles as well [45]. This occurs when the propagating signal contains frequency components above the cutoff frequency, which depends on the static compression force [46].

This work investigates the formation and propagation of nonstationary signals (quasiharmonic, solitary and shock waves) in linear, weakly nonlinear and strongly nonlinear diatomic periodic chains of particles. The influence of band-gaps on signal propagation and their tunability within the audible frequency range are investigated using chains composed of PTFE spheres and stainless steel cylinders.

\section{Experimental procedures}

In these experiments the diatomic chain consisted of a periodic arrangement of PTFE spheres and stainless steel cylinders where the masses were placed vertically in a PTFE cylinder with an inner diameter of $5 \mathrm{~mm}$ (shown in Fig. 1a).

The chain consisted of 19 PTFE spheres (McMaster-Carr) with diameter $d_{P}=2 R_{P}=4.76 \mathrm{~mm}$ and mass $m=0.1226 \mathrm{~g}$ and 18 stainless steel cylinders (McMaster-Carr) with height $h=3.12 \mathrm{~mm}$, diameter $d_{S}=4.96 \mathrm{~mm}$ and mass $M=0.485 \mathrm{~g}$. The stainless steel cylinders were used to ensure a planar geometry of the deformed contact, as assumed for Hertz contact. An additional ferromagnetic steel particle was placed on the top of the chain to magnetically induce a compression force $F_{0}=2.38 \mathrm{~N}$ which decreased the distance between the particle centers by $\delta_{o}=7.34 \mu \mathrm{m}$ (from Eqs. (2) and (3)).

Three calibrated piezoelectric sensors were embedded in two of the PTFE spheres and one stainless steel cylinder and connected to an oscilloscope Tektronix TDS 2014 (with RC of the electrical circuit $\sim 1 \mathrm{~ms}$ ) to measure the force amplitude and determine the speed of the signals. Each sensor was assembled using a lead zirconate-titanate piezoelement $\left(3 \mathrm{~mm}^{2}\right.$ plates with thickness $\left.0.5 \mathrm{~mm}\right)$ with nickel-plated electrodes and microminiature wiring embedded in the particles (Fig. 1b). The particles with these sensors were placed in the 2nd, 14th and 27th positions from the top (see Fig. 1a). The total mass of each particle including the sensor was approximately equal to the mass of the PTFE particle and stainless steel cylinder, respectively. The small mass difference $(<6 \%)$ between the particles and particles with sensors were taken into account in the numerical 
(a)

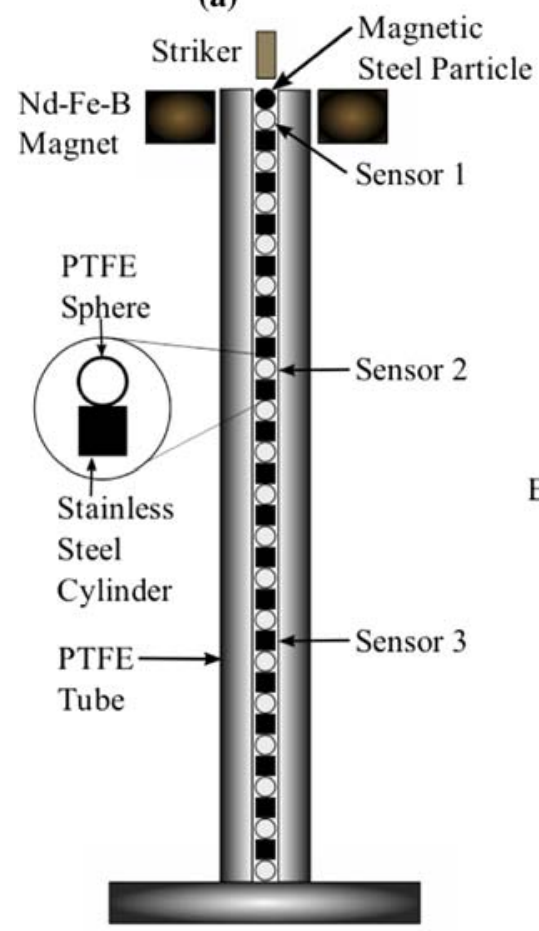

(b)

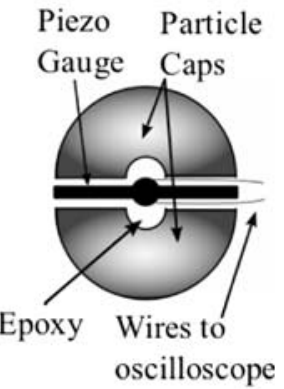

Fig. 1 Schematic diagram of the experimental setup. a The diatomic chain is composed of stainless steel cylinders and PTFE spheres. Two sensors were embedded inside PTFE particles (sensors 1,2) and one (sensor 3) inside stainless steel cylinder). A Neodymium-Iron-Boron ring magnet was used for initial static compression and an alumina striker was used for impact excitation. The inset in a shows the composition of a unit "cell" as the basis of the diatomic system. b Schematic of a PTFE particle with an embedded piezoelectric sensor

calculations and created negligible effects on the wave propagation. The sensors were calibrated using the conservation of linear momentum in separate impact experiments.

The pulses were created by impacts of four different strikers made from $\mathrm{Al}_{2} \mathrm{O}_{3}$ cylinders of different masses, comparable to or larger than the mass of one "cell" in the chain. Each cell consisted of one PTFE sphere and one steel cylinder and had a mass of $0.608 \mathrm{~g}$ (see the inset in Fig. 1a). The strikers used for the creation of a single and multiple solitary waves were: $0.61 \mathrm{~g}$ (about 1 cell mass), $1.22 \mathrm{~g}$ ( 2 cells mass) and $2.75 \mathrm{~g}$ ( 4.5 cells mass) and for shock-like pulses, $17.81 \mathrm{~g}$ (about 29 cells mass). The impact velocities used in experiments were $0.44 \mathrm{~m} / \mathrm{s}$ for the first three masses and $0.20 \mathrm{~m} / \mathrm{s}$ for the $17.81 \mathrm{~g}$ striker.

\section{Band-gap in a linear diatomic chain}

A linear elastic diatomic chain can be assembled from periodic arrangements of elements of different masses and material properties, which are initially compressed by a static force. From an experimental point of view it is convenient to employ the elastic contact of soft spherical particles (PTFE spheres) and elastically rigid cylinders (stainless steel cylinders). The contact between them results in a relatively low elastic modulus of the system leading to a frequency gap within the audible range for possible practical applications.

The diatomic mechanical system studied (PTFE spheres, stainless steel cylinders) is shown in Fig. 2. The crosses and black circles represent the initial and current positions of the centers of the corresponding masses in a statically compressed chain, $h / 2$ denotes half of the height of the cylinder, $R_{P}$ is radius of the PTFE sphere, and $\delta_{0}$ is the relative displacement of the neighboring particles due to static compression. The initial static force may be applied using magnetically induced compression [37]. The linear case presented in Fig. 2a shows that the compression due to the static force $F_{0}$ is much larger than the perturbed force in the propagating wave. Conversely, the perturbed force in the nonlinear wave is greater than or comparable to the applied force, $F_{0}$ (Fig. 2b). 

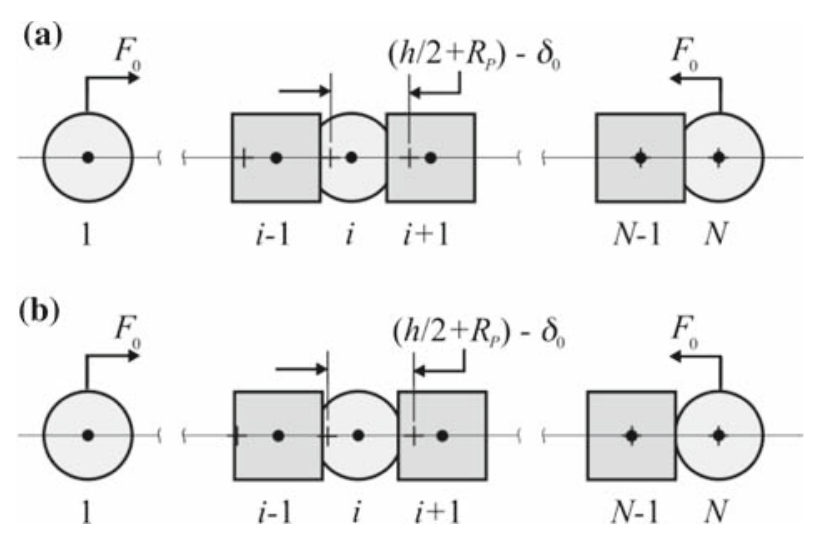

Fig. 2 Relative positions of the particles in a diatomic mechanical system corresponding to two qualitatively different wave regimes: a strong static compression (small variation of dynamic compressive forces between particles in the wave with respect to initial forces between them, the weakly nonlinear case) and $\mathbf{b}$ weak static compression (large variation of dynamic compressive forces between particles in the wave with respect to initial forces between them, the strongly nonlinear case). The relative contact area between the particles in the middle of the chain, disturbed by a propagating wave, in comparison with those in undisturbed ends indicates the different regimes of behavior. The comparison of $\mathbf{a}$ and $\mathbf{b}$ illustrates that a dramatic change of behavior of the system can be accomplished without significant changes of the overall geometry of the system

The phononic band-gap frequencies in an elastic granular chain (Fig. 2a) can be found through the linearization of the force displacement relationship between the two particles in the unit cell:

$$
F=A\left(\delta_{0}+\delta_{d}\right)^{3 / 2},
$$

where $F$ is the total compressive force including $F_{0}$ created by the static compression, and the dynamic part of the force $F_{d}$ is due to the wave disturbance. The constant $A$ depends on the elastic properties and geometry of the contacting particles [31],

$$
A=\frac{4 E_{P} E_{S}\left(1 / R_{S}+1 / R_{P}\right)^{-1 / 2}}{3\left[E_{S}\left(1-v_{P}^{2}\right)+E_{P}\left(1-v_{S}^{2}\right)\right]},
$$

where $E_{P}=1.46 \mathrm{GPa}$ [33], $E_{S}=193 \mathrm{GPa}$, and $v_{P}=0.46$ and $v_{S}=0.3$ are the elastic moduli and Poisson's ratio for PTFE and stainless steel, and $R_{S}$ (in this case $1 / R_{S}=0$ ) and $R_{P}=4.76 \mathrm{~mm}$ are the radii of contact of the cylinder with a planar contact surface and PTFE sphere. From Eq. (2) it is clear that elastic properties of PTFE with a very low elastic modulus, in comparison with steel, dominate the interaction force between the particles in the system.

Assuming $\delta_{o} \gg \delta_{d}$, a Taylor expansion may be used to linearize Eq. (1),

$$
F \approx A \delta_{o}^{3 / 2}+\frac{3}{2} A \delta_{o}^{1 / 2} \delta_{d}
$$

The two terms in Eq. (3) are the static force $F_{0}$ and the linearized form of dynamic force $F_{d}$. The two force components are $F_{0}=A \delta_{o}^{3 / 2}$ and $F_{d}=\frac{3}{2} A \delta_{o}^{1 / 2} \delta_{d}=\beta \delta_{d}$, where

$$
\beta=\frac{3}{2} A \delta_{o}^{1 / 2}=\frac{3}{2} A^{2 / 3} F_{o}^{1 / 3} .
$$

The linearized forces between particles result in two linearized equations of motion for the cylinders and spheres with displacements $u_{2 n}$ and $v_{2 n+1}$ from their equilibrium positions in the compressed chain (these equations are valid only for particles inside chain, and not for end particles),

$$
\begin{aligned}
M \ddot{u}_{2 n} & =\beta\left(v_{2 n-1}+v_{2 n+1}-2 u_{2 n}\right), \\
m \ddot{v}_{2 n+1} & =\beta\left(u_{2 n+2}+u_{2 n}-2 v_{2 n+1}\right),
\end{aligned}
$$

where $n$ are integers equal to $1,2, \ldots,(N-1) / 2$, in Eq. (5) and equal $1,2, \ldots,(N-3) / 2$, in Eq. (6), $N$ is the number of particles (odd numbers of particles coordinates correspond to spheres and even to cylinders, $N$ is an odd number according to Fig. 2), and $M$ and $m$ denote the masses of the cylinder and sphere. The derivation 


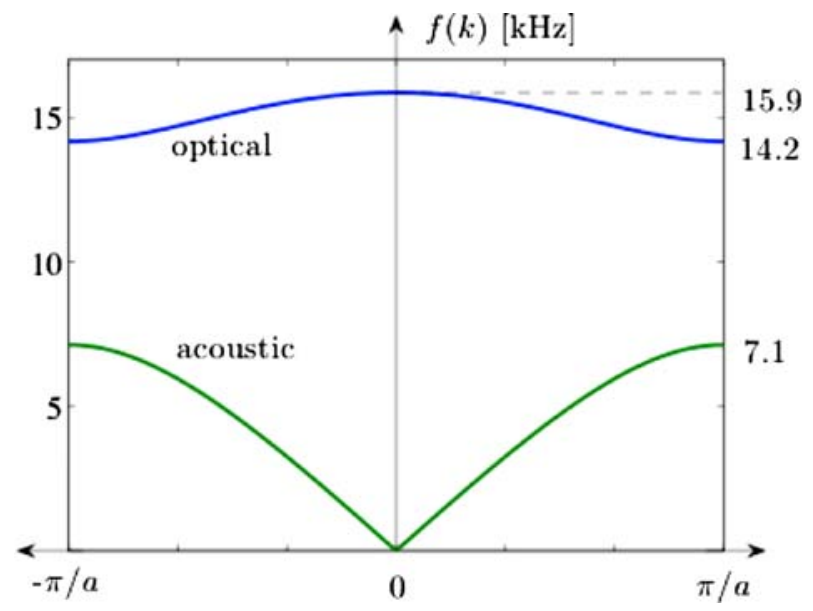

Fig. 3 Dispersion relation in the first Brillouin zone for linear elastic diatomic chain composed of stainless steel cylinders and PTFE spheres used in the experiments

below follows the classical analysis of a diatomic chain which can be found, for example, in [11]. The amplitudes of motion are obtained by substituting the propagating wave solutions represented by $u_{2 n}=u e^{i\left(k x_{2 n}-\omega t\right)}$ and $v_{2 n+1}=v e^{i\left(k x_{2 n+1}-\omega t\right)}$ into Eqs. (5) and (6) [11],

$$
\begin{aligned}
M \omega^{2} u & =2 \beta u-2 \beta v \cos (k a / 2), \\
m \omega^{2} v & =2 \beta v-2 \beta u \cos (k a / 2),
\end{aligned}
$$

where $a=2\left(h / 2+R_{P}-\delta_{0}\right)$ is the unit cell size. Equations (7) and (8) have nontrivial solutions for amplitudes of vibrating masses when the determinant is equal to zero. The dispersion relation is:

$$
\omega^{2}=\frac{\beta}{m M}\left(m+M \pm \sqrt{m^{2}+M^{2}+2 m M \cos (k a)}\right) .
$$

This dispersion relation for the linear elastic diatomic chain is shown in Fig. 3 for $f=\omega / 2 \pi$ (the plus and minus sign in Eq. (9) corresponds to the optical and acoustic branches). The lower and upper bound of the band-gap for this system are expressed by the following equations [11]:

$$
f_{1}=\frac{1}{2 \pi}\left(\frac{2 \beta}{M}\right)^{1 / 2}, \quad f_{2}=\frac{1}{2 \pi}\left(\frac{2 \beta}{m}\right)^{1 / 2} .
$$

The band-gap frequencies may be tuned by changing the value of $\beta$ that depends on $A$ and the initial compressive force $F_{0}$. The band-gap is sensitive to the material properties $E$ (Young's modulus), and $v$ (Poisson's ratio), the particle radii $R$ and the initial static compression [Eq. (2), (3)]. Note that the band-gap frequencies are nonlinearly dependent on the initial compression force $\left(f \sim F_{0}^{1 / 6}\right)$.

The corresponding elastic moduli and Poisson's ratio for the diatomic chain are $E_{P}=1.46 \mathrm{GPa}$ [33], $E_{S}=193 \mathrm{GPa}$, and $v_{P}=0.46$ and $v_{S}=0.3$, respectively. Based on Eqs. (2), (4) and (10) with $F_{0}=2.38 \mathrm{~N}$ $\left(\delta_{0}=7.34 \mu \mathrm{m}\right)$ these parameters result in the limiting frequencies $f_{1}=7,120 \mathrm{~Hz}$ and $f_{2}=14,162 \mathrm{~Hz}$ (Fig. 3) that are within the audible range from $20-20,000 \mathrm{~Hz}$.

\section{Results and discussions}

\subsection{Signal transformation in a tunable linear diatomic chain}

Signals with pulse amplitudes much smaller than the magnetically applied static force propagate in the linear regime. It is important to find the transient response of the specific linear system with the boundary conditions that are close to a harmonic excitation with frequencies within the band-gap (resulting in an evanescent wave) to identify a characteristic spatial scale where the band-gap is able to affect the shape of the propagating pulse. 

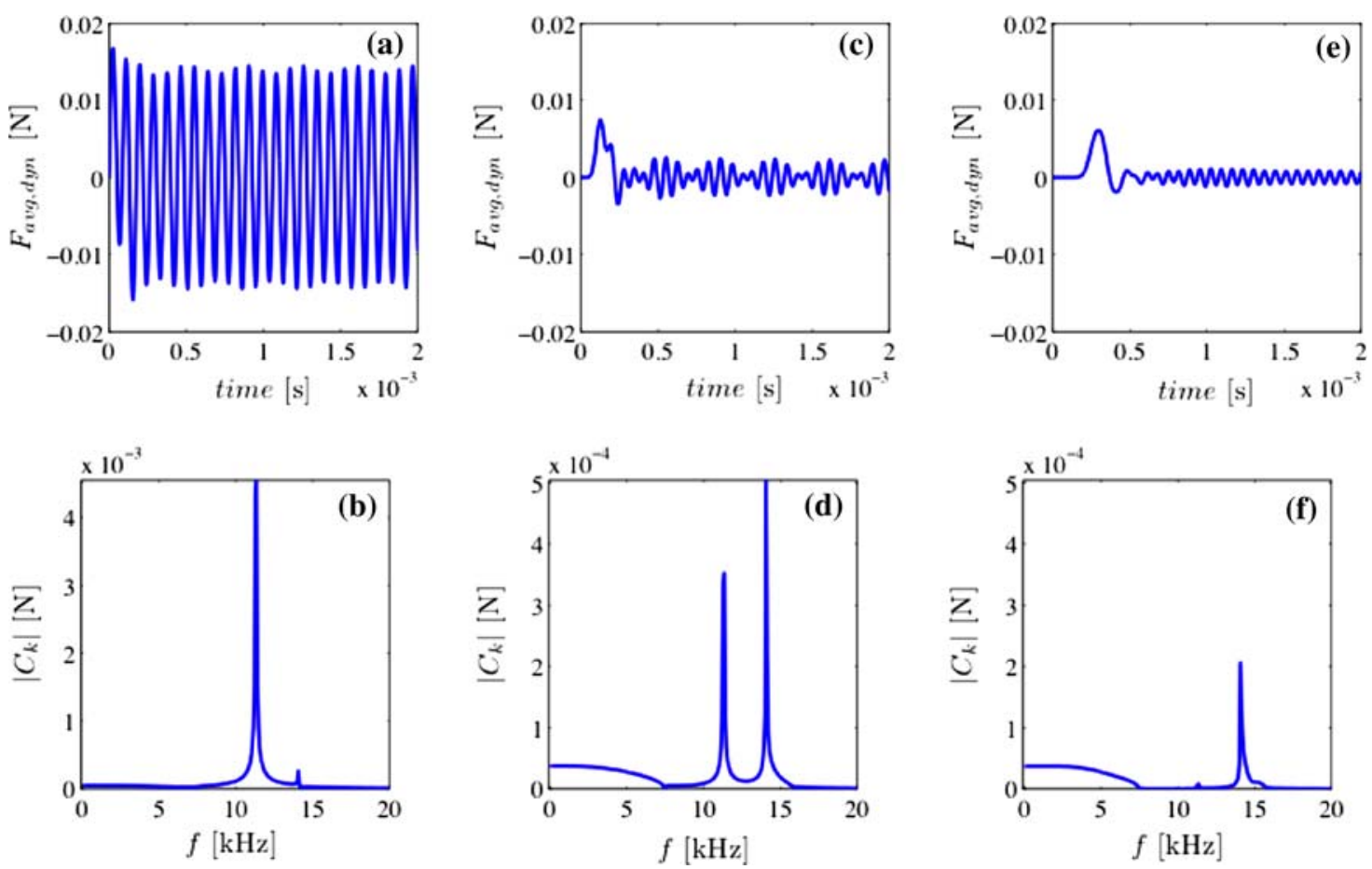

Fig. 4 Linear chain with a static force $F_{0}=2.38 \mathrm{~N}$. The top PTFE sphere was given an initial velocity of $0.00442 \mathrm{~m} / \mathrm{s}$. a Dynamic force between the top PTFE particle and stainless steel cylinder and $\mathbf{b}$ its Fourier spectrum; $\mathbf{c}$ dynamic force pulse inside 5th particle (PTFE) (averaged of contact forces between 4th (SS) and 5th (PTFE) and 5th (PTFE) and 6th (stainless steel cylinder) particles) and $\mathbf{d}$ its Fourier spectrum; $\mathbf{e}$ dynamic force in transmitted pulse inside 11th (PTFE) particle and $\mathbf{f}$ its Fourier spectrum

It should be mentioned that the existence of a band-gap does not address the question about the distance it takes for the entering signal to be affected by it.

In the linear case the pulses are transformed by dispersion [31] and the role of nonlinearity is not essential. In Figs. 4-13 the pulses and their corresponding frequency spectrum are plotted for comparison. The abscissa values of the frequency plots are the Fourier coefficients derived from a fast-Fourier-transform,

$$
C_{k}=\hat{X}(k) / M=\sum_{j=1}^{N} X(j) / M \exp [-2 \pi i(j-1)(k-1) / N],
$$

where $X(j)$ denotes the vector with length $N$ to be transformed, $j$ and $k$ increment over time and frequency and $M$ is the length of $X(j)$ without 'padded' zeros. For example, $C_{0}$ denotes the mean of the transformed function. In each of the following figures, a ratio of $N / M=5$ was used since the pulses were finite and not exactly periodic. These values were chosen for ease of correlation between the pulse and its spectrum.

In the numerical calculations an oscillatory pulse with a frequency inside the band-gap was created at one end of the periodic diatomic chain (Fig. 1) composed of 300 cells, with a PTFE sphere at the top resulting in signals with evanescent frequencies propagating in the system at the short distances from the entrance. A static force of $2.38 \mathrm{~N}$ was applied to this PTFE sphere and it was given an initial velocity of $0.00442 \mathrm{~m} / \mathrm{s}$. This resulted in an oscillatory excitation that was approximately harmonic (Fig. 4a, b). The amplitude of the dynamic force between the top PTFE sphere and the stainless steel cylinder was $0.015 \mathrm{~N}$ (160 times smaller than the initial compression force) and did not allow the top PTFE particle to separate from the chain. The majority of the frequency spectrum in Fig. $4 \mathrm{~b}$ is between the band-gap frequencies with its largest frequency component at $11 \mathrm{kHz}$. The Fourier spectrums for all cases were found for propagating pulses up to $15 \mathrm{~ms}$, but the data in Fig. 4a, c and e are truncated to clearly show the leading pulse behavior.

The numerical results of the force in the propagating pulse (i.e. the force inside 5th PTFE particle belonging to the third cell and the force inside 11th PTFE particle belonging to the sixth cell) and their frequency spectrums are shown in Fig. 4c, d, e, and f, respectively. The pulses found in numerical calculations correspond to the averaged dynamic force in Figs. 4-13 in the paper. The amplitude of the first pulse at the 6th cell is 
$0.006 \mathrm{~N}$, which is about 400 times lower than the initial compression force, and its width is about nine particles (or 4.6 cells). An estimation of the pulse width was made based on its duration and speed assuming slow transformation of its shape within the distance comparable to few cell sizes. At the 11th particle the width slightly increases to 4.7 cells. At the 21 st particle the wavelength of the propagating pulse becomes almost twelve particles (or about 5.9 cells). It is interesting that the initial wavelength is close to five cells similar to the pulse width in a strongly nonlinear diatomic chain without initial compression [31,37].

It is clear from Fig. 4 that this linear chain is able to dramatically change the shape of the initial oscillatory pulse within relatively short distances from the top PTFE particle (within only six cells!), which is mainly due to dispersion. As the signal propagates, the frequency spectrums in Fig. $4 \mathrm{~b}, \mathrm{~d}$ and $\mathrm{f}$ show that the pulse preferentially transforms (demodulates) such that the frequency components within the band-gap limits move toward lower frequencies.

The Fourier spectrum of the force in the system very close to the top (inside the 5th PTFE particle, Fig. 4c, d) demonstrates significant frequency components above and below the band-gap in addition to the initial signal. This is significantly different from the spectrum of the excited pulse (Fig. 4a, b). There are two main frequencies in Fig. 4d: one is the source signal at about $11 \mathrm{kHz}$ and the other is about $14 \mathrm{kHz}$, which was also present in the input signal at about the same amplitude. These two harmonics are responsible for the beating phenomenon observed in the force signal (Fig. 4c). This beating disappears and the trailing oscillations become approximately harmonic at the 11th particle (PTFE) (Fig. 4e) due to the complete transformation of the $11 \mathrm{kHz}$ frequency component.

It should be mentioned that the high frequency corresponding to the top of the band-gap does not disappear during signal propagation. This is due to the oscillatory motion of the light PTFE particle in the cell, which is also a characteristic of nonlinear signals [31, Chap. 1, Fig. 1.19]. The relatively small peak around $14 \mathrm{kHz}$ in Fig. $4 \mathrm{~b}$ is present in the initial signal due to the motion of top PTFE particle against the steel cylinder under constant force. The amplitude of this frequency component decays very slowly (compare Figs. $4 \mathrm{~b}, \mathrm{~d}$ and f) in striking contrast to transformation of the initial $11 \mathrm{kHz}$ component.

It is interesting to investigate pulse transformation at larger distances from the top particle to investigate the behavior of the leading pulse. The shapes of the pulses are presented in Fig. 5a for the 51st, 101st and 151st particle. In Fig. 5a the pulse continues to change due to linear dispersion; particularly the leading pulse is not of solitary shape at these distances from the top particle as it appears closer to the entrance (Fig. 4c, e). The leading part of the propagated signal measured at the $101 \mathrm{st}$ particle up to $7 \mathrm{~ms}$ has a broad band of frequencies below the band-gap (curve 1 in Fig. 5b), and after $7 \mathrm{~ms}$ the signal is characterized by one peak at the bottom and top of the band-gap (curve 2 in Fig. 5b). It should be mentioned that the harmonic with frequency about $14 \mathrm{kHz}$ continues to decay with the pulse propagation (compare Fig. 4d, f with Fig. 5b, curve 2). The wave packet containing frequencies at the top of the band-gap (not shown on the bottom wave profile in Fig. 5a) propagates with a lower speed in comparison with the leading oscillatory pulse composed of frequencies lower than $7 \mathrm{kHz}$. It should be mentioned that there is no stationary single pulse in the linear dispersive case, and the leading pulse will evolve further during propagation (e.g., with decreasing amplitude and increasing width), but the main features of it are already established before it reaches the 50th cell.

\subsection{Signal transformation in a nonlinear diatomic chain}

The band-gap is a property of linear systems [Eqs. (7), (8)] and it is not clear if it can influence the signal propagation in the nonlinear regime. Recently, the effects of varying isotropic static compression in the linear and nonlinear regimes for triangular (two-dimensional) lattices of identical steel spheres show evidence of selfdemodulation in the time-frequency plots of acoustic signals [46]. Here, the behavior of signals in diatomic chains with amplitudes comparable to the initial compression is investigated numerically.

A nonlinear pulse in the same diatomic chain as in the previous section was created when the top PTFE particle was given a higher initial velocity of $0.442 \mathrm{~m} / \mathrm{s}$ (Fig. 6a, b), which is two orders of magnitude larger than in the linear system, though the particles in the chain remain in contact. This resulted in a similar oscillatory excitation shown in Fig. 4a and b, but the dynamic forces between the top PTFE sphere and stainless steel cylinder have an amplitude comparable to the static compression force (being 1.6 times smaller). The numerical results of the force in the pulse propagating inside the system and their frequency spectrums are shown in Fig. 6c, d, e, and f, respectively. The force inside the 5th PTFE particle belongs to the third cell and the force inside the 11th PTFE particle belong to the sixth cell. The amplitude of the first pulse at the 6th cell is $0.65 \mathrm{~N}$ and it is about 3.7 times lower than the initial compression force, and its width is about nine particles 

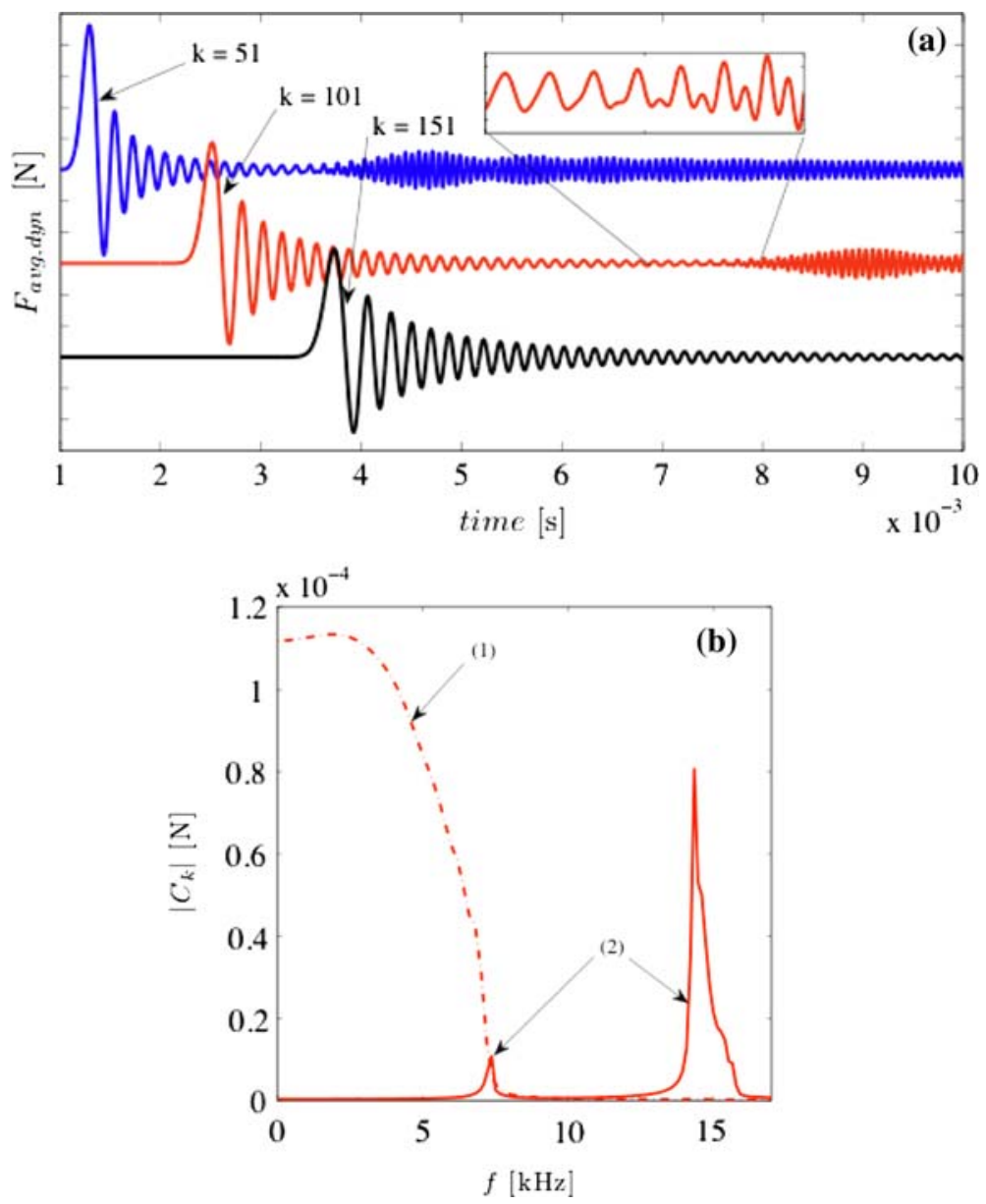

Fig. 5 Linear chain, static force $F_{0}=2.38 \mathrm{~N}$, the top PTFE sphere was given an initial velocity of $0.00442 \mathrm{~m} / \mathrm{s}$. a Forces in the transmitted pulses inside 51st particle ( $k=51$, PTFE) (averaged of contact forces between 50th (stainless steel cylinder) and 51st (PTFE) and 51st (PTFE) and 52nd (stainless steel cylinder) particles, inside the 101st particle $(K=101)$ and inside 151 st particle $(K=151)$. The vertical scale is $0.001 \mathrm{~N} / \mathrm{div}$ and the three curves were offset for visual clarity. The inset in a shows the transition from acoustic to optical modes in the 101st particle. b Curve (1): the Fourier spectrum for the leading pulse for 101st particle up to the time $7 \mathrm{~ms}$ with mainly acoustic frequency; Curve (2) is the Fourier spectrum for the trailing wave group from the time 7 to $12 \mathrm{~ms}$ containing the optical frequency component

(or 4.6 cells). This pulse is widening when it propagates in the system. For example at the 21 st particle the width is approximately eleven particles (or about 5.7 cells), which is closer to the width of a stationary pulse in strongly nonlinear diatomic chains without static compression [31,37].

At relatively short distances from the impacted end (about six cells) it is clear that this nonlinear chain dramatically changes the shape of the pulse as its frequency spectrum shifts toward lower and higher frequencies. The behavior of the signal in this nonlinear chain is similar to the case of a linear chain, suggesting that the nonlinearity of the chain does not influence the signal propagation as much as dispersion. This means that, for practical applications, the influence of the band-gap is also relevant for finite amplitude nonlinear signals within short distances from the signal source.

The pulse transformation as it propagates into the system at larger distances from the impacted end (up to the 151st particle) is shown in Fig. 7. As in the linear case (Fig. 5) the leading propagating pulse continues to transform due to dispersion even in the presence of nonlinearity. However, at much larger distances, the leading solitary wave, which is a steady solution in the weakly nonlinear case, may separate from the oscillatory part of the signal in contrast to the linear case.

At the 101st particle the leading oscillating pulse (up to $7 \mathrm{~ms}$ ) has a broad band of frequencies below the band-gap (curve 1, Fig. 7b), and for the interval from $7 \mathrm{~ms}$ to $12 \mathrm{~ms}$ it is characterized by two peaks at the vicinity of the bottom $\left(f_{1}\right)$ and one at the top of the band-gap $\left(f_{2}\right)$ (curve 2, Fig. 7b). In contrast to Fig. 5b 

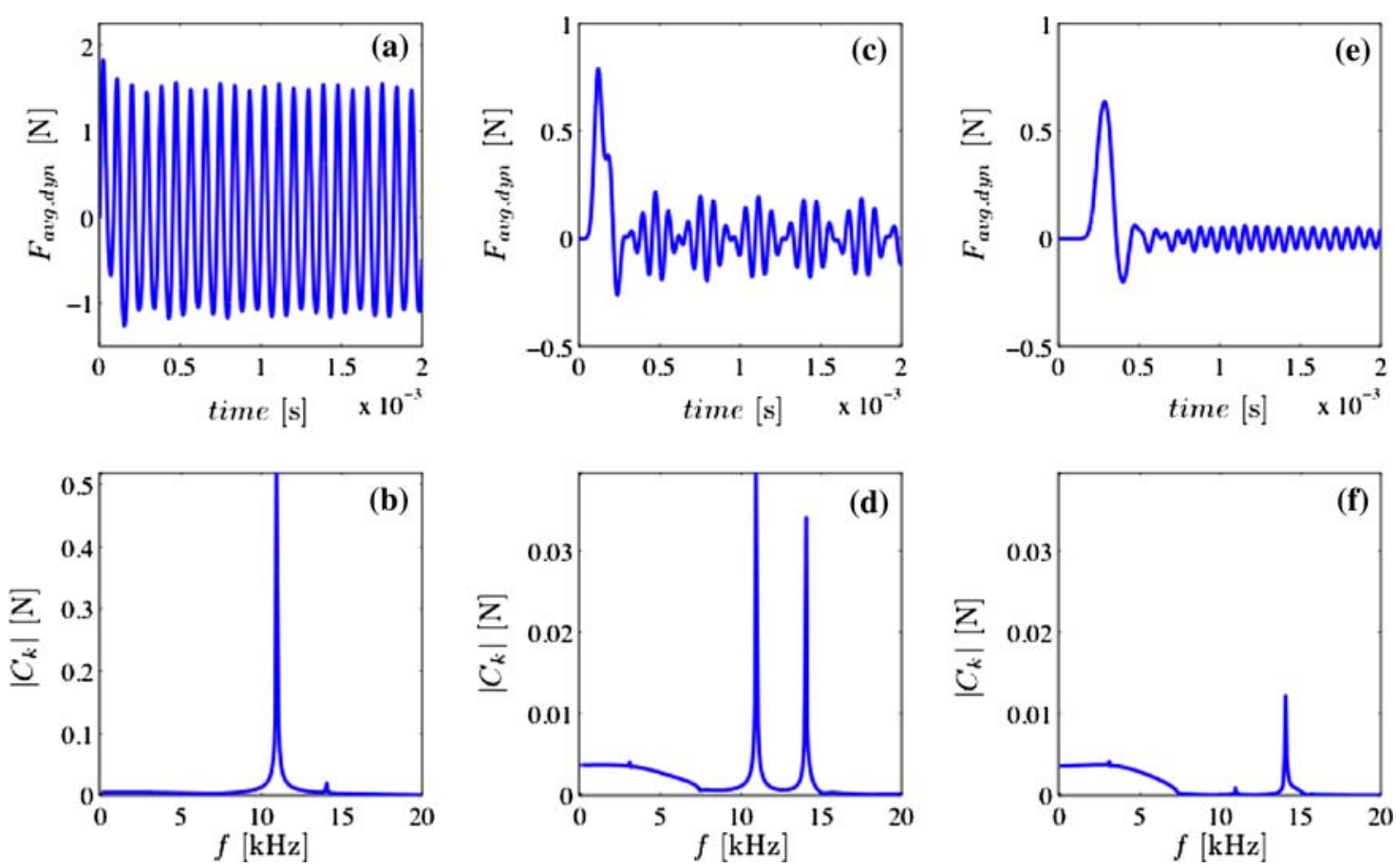

Fig. 6 Nonlinear chain with a static force of $F_{0}=2.38 \mathrm{~N}$ applied to the top PTFE sphere, which was given an initial velocity of $0.442 \mathrm{~m} / \mathrm{s}$. a The dynamic force, averaged between the top PTFE particle and stainless steel cylinder (incoming pulse) and $\mathbf{b}$ its Fourier spectrum; $\mathbf{c}$ the dynamic force in the transmitted pulse inside the 5th particle (PTFE) (averaged of contact forces between 4th (SS) and 5th (PTFE) and 5th (PTFE) and 6th (stainless steel cylinder) particles and $\mathbf{d}$ its Fourier spectrum; e the dynamic force in the transmitted pulse inside the 11th (PTFE) particle and $\mathbf{f}$ its Fourier spectrum

corresponding to the linear case there is an additional $3 \mathrm{kHz}$ component, which may be due to the influence of nonlinearity in the system. The wave packet containing frequencies above $14 \mathrm{kHz}$ (not shown on the bottom wave profile) propagates with a lower speed than the leading oscillatory pulse composed of frequencies lower than $7 \mathrm{kHz}$. The oscillations of the first (compressed) particle have a harmonic at $\sim 14 \mathrm{kHz}$ (small peak in Fig. 6b), which results in signals that propagate into the system with a similar frequency and with a low speed along with the leading pulses that travel with speeds corresponding to the acoustic branch.

Since the gap in the frequency spectrum is a property of a linear elastic system it is surprising that in the nonlinear chain, which is a qualitatively different system (e.g., it supports a solitary wave absent in a linear case), the signal transformation is similar to the linear chain. In the trailing wave group we have no component inside band-gap, only components at the top of acoustic and bottom of optical bands. The frequencies at the top and the bottom are long living in comparison with those inside the band-gap in both linear and nonlinear chains. So, waves with finite amplitude in a nonlinear material are sensitive to the same range of band-gap frequencies as waves with infinitesimally small amplitudes in linear chains. This allows the tunability of the band-gap in the nonlinear range of material response based on Eq. (10) derived for an infinitesimally small amplitude of the signals.

To compare experimental results with the numerical investigation for the nonlinear chain four separate experiments were performed in a diatomic chain composed of 37 elements (including magnetically induced static compression and the gravitational preload) with strikers having different masses and velocities: 0.61, 1.22 and $2.75 \mathrm{~g}$ alumina strikers at $v_{0}=0.44 \mathrm{~m} / \mathrm{s}$ and a 17.81 -g striker at $v_{0}=0.2 \mathrm{~m} / \mathrm{s}$. Each striker generated incoming pulses of different lengths and amplitudes. In each case, the static compression was $2.38 \mathrm{~N}$, applied to the top magnetic spherical particle (in previous numerical calculations the force was applied to the top PTFE particle to generate practically harmonic boundary conditions). The experimental set up is shown in Fig. 1 and experimental data are presented in Table 1 . There is a reasonably good agreement between pulse velocities in experiments and numerical calculations within the experimental error.

The results of the impact by the 0.61-g striker are shown in Fig. 8. The impact resulted in a single pulse at the top of the chain since the striker mass was close to the mass of one cell in the system. The initial signal 

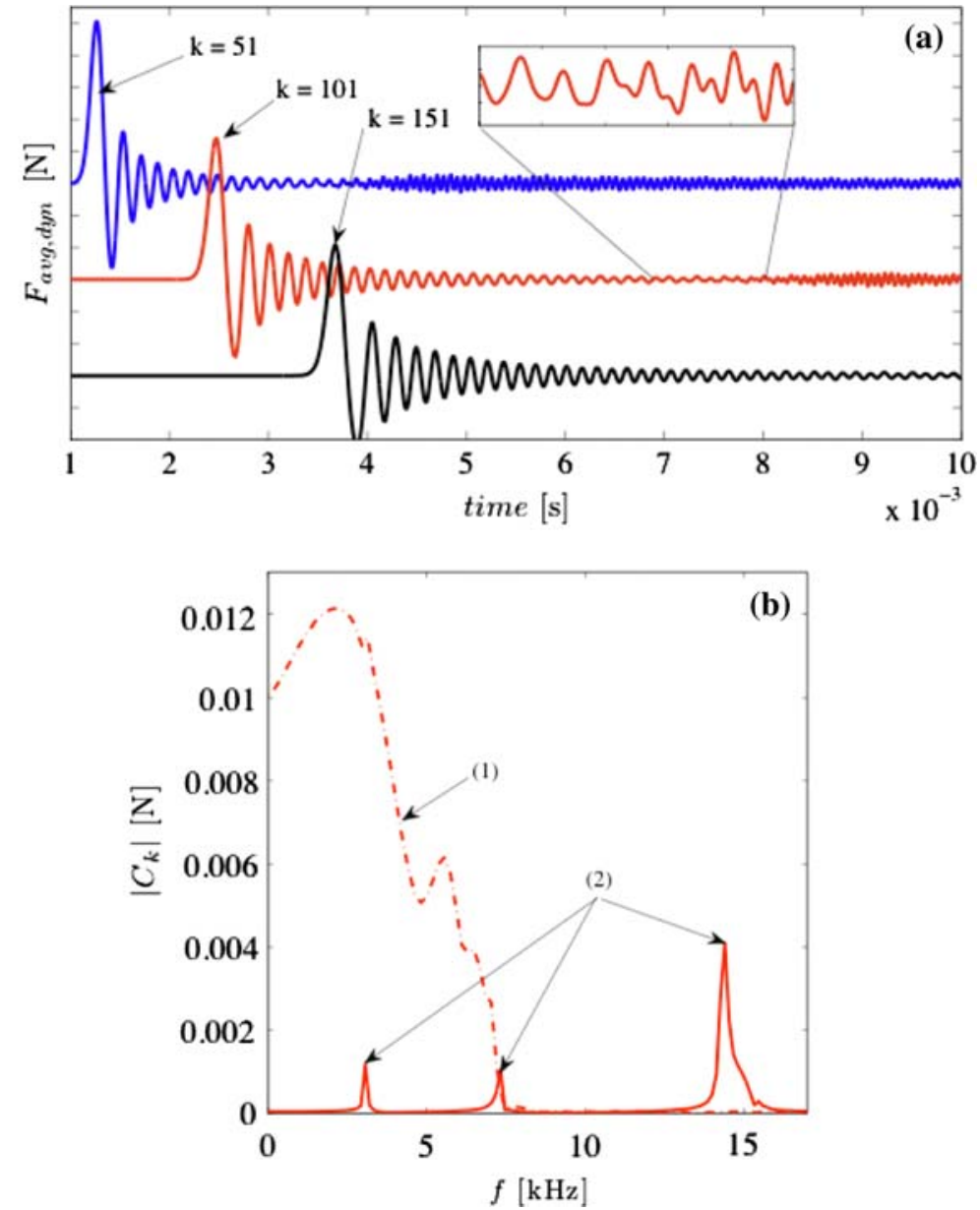

Fig. 7 Nonlinear chain with a static force of $F_{0}=2.38 \mathrm{~N}$ applied to the top PTFE sphere, which was given an initial velocity of $0.442 \mathrm{~m} / \mathrm{s}$. a Forces in the transmitted pulses inside the 51st particle ( $k=50$, PTFE) (averaged of contact forces between 50th (stainless steel cylinder) and 51st (PTFE) and 51 th (PTFE) and 52nd (stainless steel cylinder) particles, inside 101st particle $(K=101)$ and 151 st particle $(K=151)$. The vertical scale is $0.1 \mathrm{~N} /$ div and the three curves were offset for visual clarity. The inset in a shows the transition from acoustic to optical modes in the 101st particle. b Curve (1): Fourier spectrum for the leading pulse for the 101st particle, up to $7 \mathrm{~ms}$ containing acoustic frequencies; Curve (2) is the Fourier spectrum for the trailing wave group (taken from $7 \mathrm{~s}$ to $12 \mathrm{~ms}$ ), containing acoustic and optical frequencies

Table 1 Comparison of average speed $V_{s}$ of leading pulses taken between particles number 14th and 27th for experiments and numerical results in the nonlinear chain for different masses and velocities of strikers

\begin{tabular}{|c|c|c|}
\hline & $\begin{array}{l}\text { Striker mass } \\
(\mathrm{g})\end{array}$ & $\begin{array}{l}\text { Pulse speed } \\
(\mathrm{m} / \mathrm{s})\end{array}$ \\
\hline \multirow[t]{4}{*}{ Experimental } & 0.61 & 175 \\
\hline & 1.22 & 177 \\
\hline & 2.75 & 181 \\
\hline & 17.81 & 179 \\
\hline \multirow[t]{4}{*}{ Numerical } & 0.61 & 171 \\
\hline & 1.22 & 173 \\
\hline & 2.75 & 176 \\
\hline & 17.81 & 169 \\
\hline
\end{tabular}

was quite different (due to the boundary conditions) from Fig. 6a although the impulse in experiments also transformed into a single leading pulse as in Fig. 6c, e.

Figure 8 shows the experimental results (Fig. 8a) and the corresponding numerical calculations (Fig. 8c) as well as the Fourier spectrum of each in Fig. 8b, d. It is clear from Fig. 8b, d that the frequency spectrum of the initial disturbance is inside the band-gap (7.1-14.2 kHz). It is also clear that the signal is shifted toward lower 

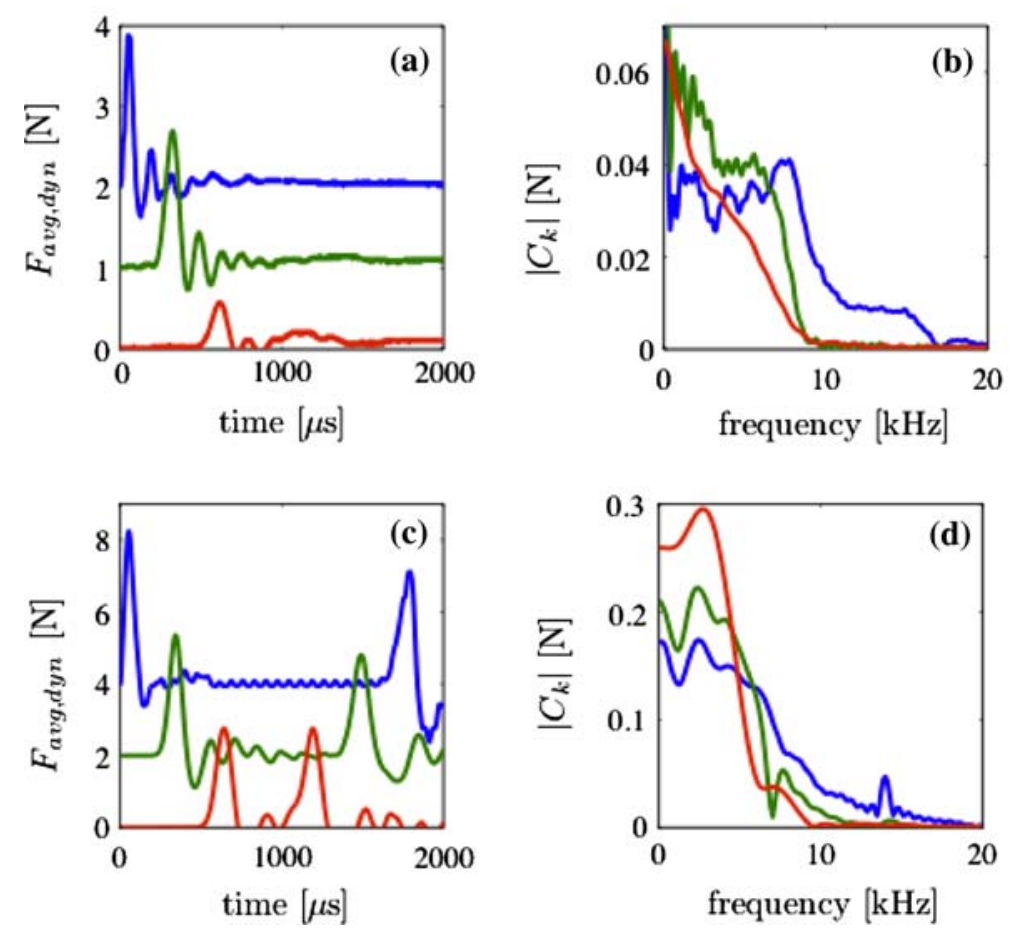

Fig. 8 Nonlinear chain with a static force of $F_{0}=2.38 \mathrm{~N}$ applied to the top magnetic particles impacted by an alumina striker with an initial velocity of $0.44 \mathrm{~m} / \mathrm{s}$. a Dynamic force in experiments recorded in the first PTFE particle (incoming pulse) and in sensors placed in the PTFE particle in the seventh cell and in the steel cylinder in the 13th cell, respectively. The curves are offset by $1 \mathrm{~N}$ for visual clarity. b Fourier spectrum for forces presented in a. c Dynamic force in numerical calculations corresponding to experimental conditions in a. The curves are offset by $2 \mathrm{~N}$ for visual clarity. $\mathbf{d}$ Fourier spectrum for forces presented in $\mathbf{c}$

frequencies within 7 cells from the impacted end. The dynamic compression of the chain effectively shifts the lower boundary of band-gap toward a higher frequency $\sim 9 \mathrm{kHz}$ than the predicted lower limit of $7.1 \mathrm{kHz}$ corresponding to static precompression (in situ modification of band-gap) explaining higher frequencies in the propagating pulse than expected in comparison with the linear case. This fast modification of the signal is similar to the previously observed for practically harmonic incoming excitations in linear and nonlinear cases (Figs. 4 and 6b, d, f). It should be mentioned that a similar behavior of the Fourier spectrum corresponding to numerical calculations and to experimental data (specifically the same cut-off frequency about $9 \mathrm{kHz}$ ) suggests that dissipation (apparently affecting the amplitude of the signal in experiments) is not the main cause for the observed differences in the frequency spectrum of each sensor.

We consider this as a direct influence of the band gap. This point is supported by the fact that continuing attenuation of the pulse amplitude in experiments did not result in a change of the cut-off frequency (compare green and red lines in Fig. 8a, b). This behavior agrees well with the transformation of the spectrum in numerical calculations presented in Fig. 8d, where dissipation is not included. We consider that experimental results are sufficient to make the statement that band gap effects determine the transformation of pulses in our cases, especially revealed in the cut-off frequency. We also would like to emphasize that the cut-off frequency of this system is inside the audible range of frequencies which may be important for some applications.

Based on numerical calculations (Fig. 4), and assuming exponential decay, the characteristic length of wave attenuation (1/e distance) for the evanescent wave in the band gap is found to be about 1 cell size, and it is practically the same for linear (Fig. 4) and nonlinear (Fig. 6) waves. If we assume that the typical space scale for attenuation of harmonics with frequencies inside the band gap and in the vicinity of it due to dissipation is similar, then the estimation of the 1/e distance from experimental data for the pulse amplitude gives us about 70 cell sizes at the beginning of the attenuation where the major modification of the spectrum takes place (Fig. 8b, compare blue and green curves). The difference in these scales also supports our point that the main input in signal transformation in experiments is due to the band gap effect and not to dissipation. Regardless of the mechanism of signal transformation, whether it is due to dissipation, dispersion, nonlinearity, discreteness, we observe that the cutoff frequency coincides with the value predicted from the linearized chain. 

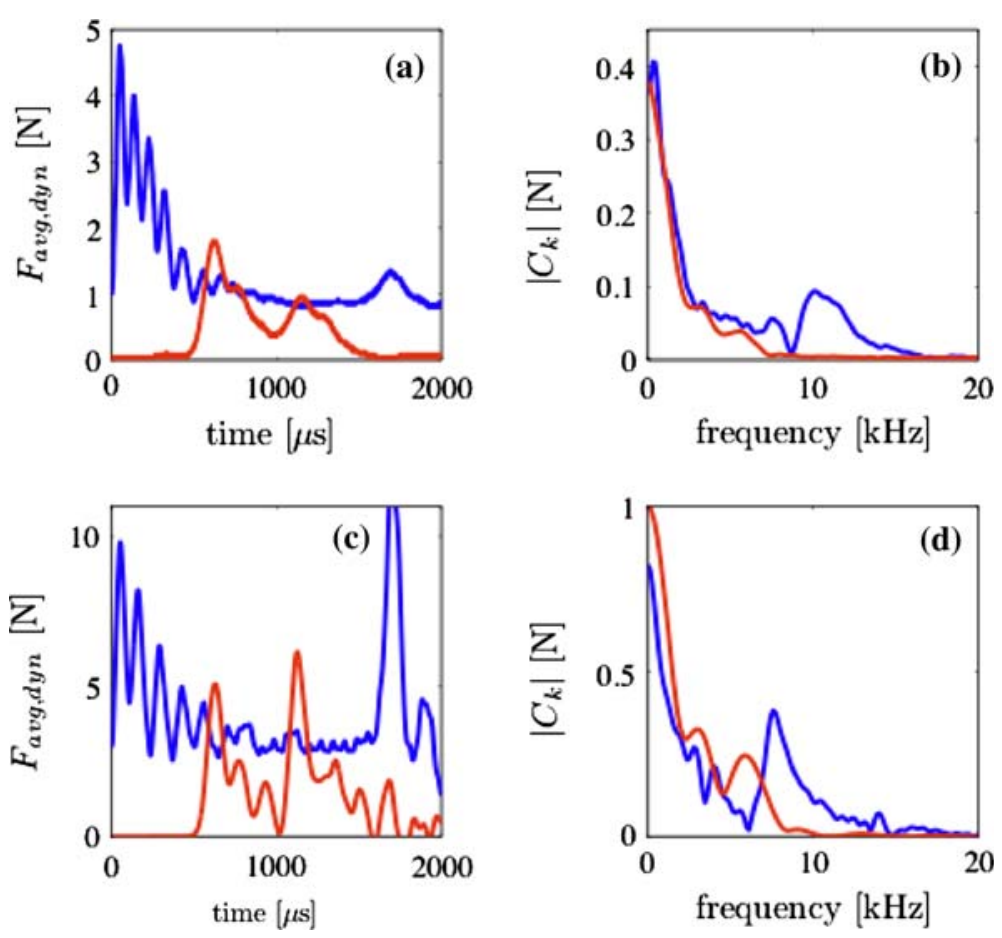

Fig. 9 Nonlinear case, static force $F_{0}=2.38 \mathrm{~N}$. The striker had a mass equal to 4.5 cells $(2.75 \mathrm{~g})$ and an initial velocity of $0.44 \mathrm{~m} / \mathrm{s}$. a Dynamic force in experiments between first PTFE particle and stainless steel cylinder (incoming pulse) and between sensors in the steel cylinder in the 13th cell and in the wall, respectively; b Fourier spectrum for forces presented in a; $\mathbf{c}$ Dynamic force in numerical calculations corresponding to experimental conditions in $\mathbf{a} ; \mathbf{d}$ Fourier spectrum for forces presented in $\mathbf{c}$

A similar behavior of the signals in the frequency domain both in experiments and numerical calculations was observed for pulses excited by the impact of an alumina striker $(1.22 \mathrm{~g}$ at $0.44 \mathrm{~m} / \mathrm{s})$ generating a series of pulses.

To increase the duration and amplitude of the incident signal, experiments were conducted using an alumina striker of larger mass $(2.75 \mathrm{~g}$ at $0.44 \mathrm{~m} / \mathrm{s})$. In Fig. 9 the experimental and numerical results of the impact of this striker are shown. This impact created a sequence of connected pulses in experiments, and numerical calculations and the corresponding frequency spectrum of the incoming pulses are shown in Fig. 9b and d. The frequency spectrum shows that the initial pulse corresponding to the force inside the second (PTFE) particle has a significant portion within the band-gap. In comparison with Fig. 8 the main portion of the frequency spectrum within the band-gap extends to a slightly higher frequency. This may be due to an additional effect of a larger mean effective dynamic compression in addition to the static force and thus increases the lower limit of the band-gap (in situ modification of band-gap). From a comparison of the data for the 2nd and 27th (PTFE) particle it is clear that the spectrum has shifted to lower frequencies; the spectrum for the pulse reflected from the wall is not shown for clarity of the graphs. In experiments and numerical calculations the spectrum cut-off observed for the 27 th particles is slightly higher than the predicted lower limit of the band-gap from the linear approach $(7.1 \mathrm{kHz})$.

A comparison between experimental and numerical data reveals smaller time intervals between peaks in experiments (compare Fig. 9a-c) resulting in a component of the Fourier spectrum with higher frequency than in corresponding numerical calculations (compare Fig. 9b-d). We explain this behavior by a delayed separation of oscillations in experiments due to dissipation. Nonlinear viscoelastic dissipation is considered in $[47,48]$ for a binary collision between particles and for the waves propagating in the chain.

To create an oscillatory shock-like wave (a nonsymmetric pulse with relatively short rise time and long duration of decay), in the experiments we used an impact of an alumina striker with a mass of $17.81 \mathrm{~g}$ at $0.2 \mathrm{~m} / \mathrm{s}$. In Fig. 10 the experimental and numerical results corresponding to the impact of this striker and the corresponding frequency spectrum of the pulses are shown (Fig. 10b, d).

The frequency spectrum shows that the initial pulse in the second (PTFE) particle has a significant portion within the band-gap. However, optical modes are not excited in this case. To check if this mode appears as the signal propagates the numerical calculations were extended up to $15 \mathrm{~ms}$, but the high frequency component (at about $14 \mathrm{kHz}$ ) corresponding to the optical mode trailing the leading shock wave was not observed. This 

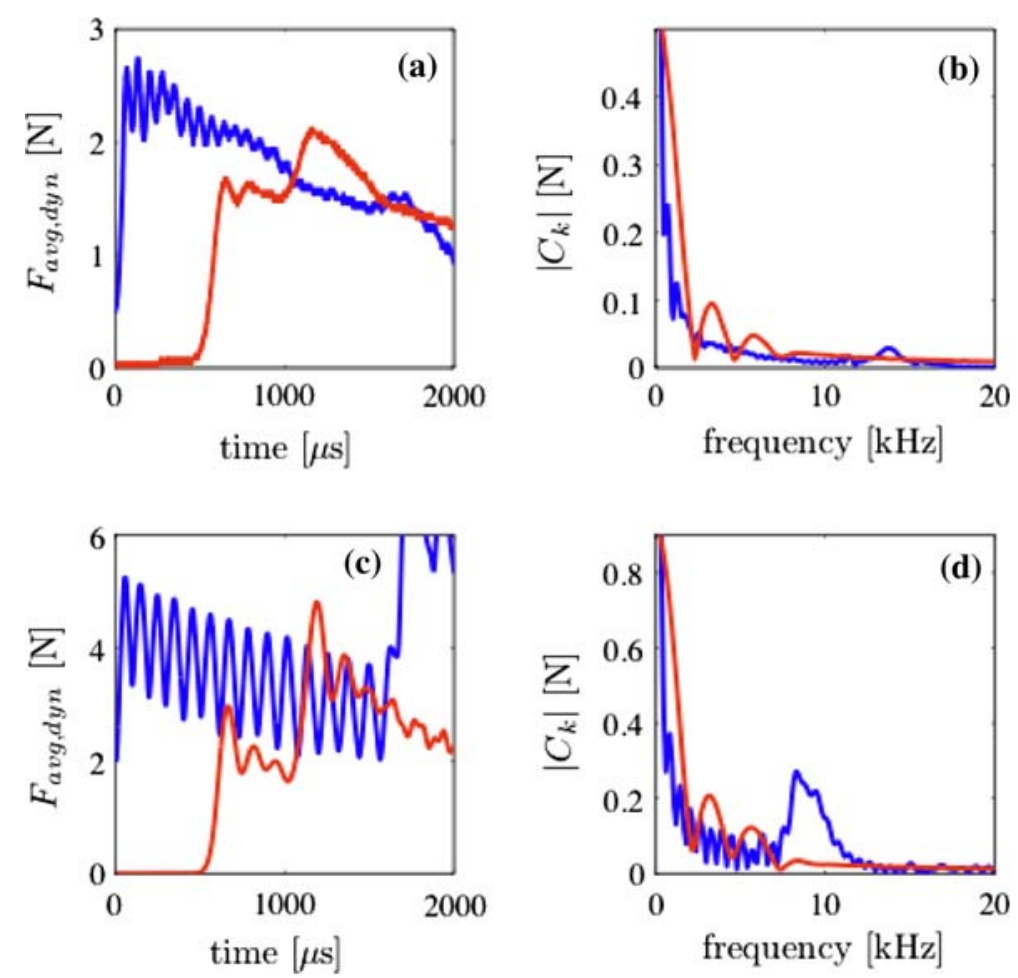

Fig. 10 Oscillatory shock waves excited in the nonlinear case in a diatomic chain composed of 37 elements, static force $F_{0}=2.38 \mathrm{~N}$. The striker had a mass equal to $17.81 \mathrm{~g}$ (about 29 cells mass) and an initial velocity of $0.2 \mathrm{~m} / \mathrm{s}$. a Dynamic force in experiments in the first PTFE particle and between sensors in the PTFE particle in the 7th cell and in the steel cylinder in the 13th cell and in the wall, respectively; $\mathbf{b}$ Fourier spectrum for forces presented in $\mathbf{a} ; \mathbf{c}$ dynamic force in numerical calculations corresponding to experimental conditions in $\mathbf{a} ; \mathbf{d}$ Fourier spectrum for forces presented in $\mathbf{c}$

contrasts the linear case with practically harmonic boundary conditions evolving into a leading pulse with an oscillatory tail (Fig. 4e, f). It may be concluded that for all shock loaded cases the high frequency component is not excited at the boundary.

In comparison to Fig. 9 the main portion of the frequency spectrum within the band-gap is centered about a slightly higher frequency in numerical calculations $(7.7 \mathrm{kHz}$ in Fig. $9 \mathrm{~d}$ and $8.4 \mathrm{kHz}$ in Fig. 10d, correspondingly). This may be due to the effect of a compressive dynamic force creating a larger mean effective precompression resulting in a higher effective stiffness of the compressed contacts in comparison with the initial state.

The oscillations in the initial shock in experiments are significantly damped in the 27th (PTFE) particle and the spectrum shifted to lower frequencies. This can be caused by dissipation if it is larger than some critical value. It was shown that dissipation is able to introduce qualitative changes in solitary and shock wave propagation in strongly nonlinear systems [48-51]. It is interesting and unexpected that "damping" of oscillations was also the case in the numerical calculations despite the fact that dissipation was not included. We explain the mechanism of the effective smoothing of the oscillating shock profile without dissipation in numerical calculations by the influences of the band-gap that eliminate the corresponding harmonic in the incoming pulse centered at about $9 \mathrm{kHz}$ within the band-gap. The effective compression in the shock wave shifts the lower boundary of the band-gap toward a higher frequency $\sim 9 \mathrm{kHz}$ than the predicted lower limit of $7.1 \mathrm{kHz}$ (in situ modification of the band-gap). Although the initial impact creates an oscillating shock, as in one mass chain $[31,49,52]$, these oscillations cannot propagate due to the band-gap. This phenomenon can be used for the design of tunable structures/metamaterials for protecting against impact loading. Comparison of two-mass chains with the same macroscopic properties (linear density and elastic constant), but different ratio between masses, was considered with respect to protection against impact [31].

Despite the fact that both experimental and numerical incoming profiles were initially oscillating, the frequencies of oscillations were quite different with a higher frequency being observed in experiments ( 9 and $12 \mathrm{kHz}$, respectively). The mechanism of generating higher frequencies in experiments can be due to slower separation of the pulses in the presence of dissipation effectively increasing the observed frequency. To check 
Table 2 Comparison of average amplitude and speed $V_{s}$ of leading pulses taken between the 14th and 27th particles for experiments and numerical results in a strongly nonlinear diatomic chain

\begin{tabular}{|c|c|c|}
\hline & $\begin{array}{l}\text { Striker mass } \\
(\mathrm{g})\end{array}$ & $\begin{array}{l}\text { Pulse speed } \\
(\mathrm{m} / \mathrm{s})\end{array}$ \\
\hline \multirow[t]{4}{*}{ Experimental } & 0.61 & 118 \\
\hline & 1.22 & 121 \\
\hline & 2.75 & 122 \\
\hline & 17.81 & 115 \\
\hline \multirow[t]{4}{*}{ Numerical } & 0.61 & 134 \\
\hline & 1.22 & 141 \\
\hline & 2.75 & 146 \\
\hline & 17.81 & 138 \\
\hline
\end{tabular}

this hypothesis viscous dissipation was included in numerical calculations (similar to [49,52]) resulting in harmonics with higher frequency (their frequency increasing with viscosity) in comparison to calculations without dissipation. It should be mentioned that magnetorheological liquids allow effective tunability of viscosity with the magnetic field [53], which can be used to tune the shock response of a granular system immersed in such media.

If the amplitude of the signal is significantly larger than the initial static compression, qualitatively new features can be expected in diatomic chains as compared to linear and nonlinear pulses. It should be mentioned that in an initially uncompressed chain (sonic vacuum) there is no characteristic band-gap that is representative of a linear elastic system. At the same time the compressed state behind a relatively long "shock wave" can exhibit a phenomenon caused by an in situ band-gap where the properties depend on the average compression caused by the wave. This was seen in the previous section for nonlinear impulses (Figs. 9, 10).

The propagation of strongly nonlinear waves in diatomic chains is not directly comparable to the previous two cases since the separation of particles is now possible with the absence of initial compression. This also occurs when the initial impact velocity value is large enough for the particle to overcome the static force. Two mass systems were investigated in this regime with a goal to mitigate impact type loading using the band gap effect.

The frequency spectrum of the propagating compressive signal should not be affected by the band-gap (Eq. 10), since both $f_{1}$ and $f_{2}$ are very small due to very weak gravitational compression in a vertical chain. In the case of impact by a particle with a mass close to the mass of the cell a single solitary pulse will have a frequency spectrum close to the solution to the long-wave approximation given in $[31,43,44]$. Higher amplitudes of strongly nonlinear solitary waves result in higher frequencies since the spatial size does not depend on the amplitude, but the signal speed increases with the wave amplitude. Thus, the allowed frequencies of harmonics composing the single solitary wave are tuned by the amplitude of the wave.

It is interesting to investigate this case because it will elucidate the effect that the length of the impact has on the resulting frequency spectrum. It was seen in the previous nonlinear case that the additional dynamic compression in the pulse may shift the initial frequency spectrum to higher frequencies.

To compare experimental with numerical results for the strongly nonlinear chain four separate experiments with an identical set up as in the previous sections (Fig. 1) were performed with the exception of the magnet used to induce static compression in the previous cases. The only static force in this case is due to gravitational preload in experiments and calculations. Each experiment has incoming pulses of different lengths and amplitudes depending on the mass of the striker to excite different Fourier spectrums and observe their evolution. The results of the experiments for a chain of PTFE spheres and steel cylinders in a strongly nonlinear regime are shown in Figs. 11, 12, 13 and Table 2. There is a reasonably good agreement between pulse velocities in experiments and numerical calculations despite dissipation present in experiments.

The mass of the striker in relation to the mass of the cell roughly corresponds to the number of significant pulses observed in experiments. For example, the impact generated by strikers $\left(\mathrm{Al}_{2} \mathrm{O}_{3}\right.$ cylinder) with masses equal to the mass of one cell (PTFE sphere and steel cylinder), 2 and 4.5 cells generated the one, two and five pulses.

In Fig. 11 the experimental and numerical results of the impact of a PTFE striker $(0.62 \mathrm{~g}$ at $0.44 \mathrm{~m} / \mathrm{s})$ are shown. This impact created one main pulse in experiments, and numerical calculations and the corresponding frequency spectrum of the incoming pulses is shown in Fig. 11b and d. In Fig. 11a, the time duration of the leading solitary wave measured in particle 14 was $330 \mu \mathrm{s}$. The average speed of its propagation between particles 14 and 27 was $118 \mathrm{~m} / \mathrm{s}$. These values result in a solitary wave width equal to 4.6 cells. This agrees 

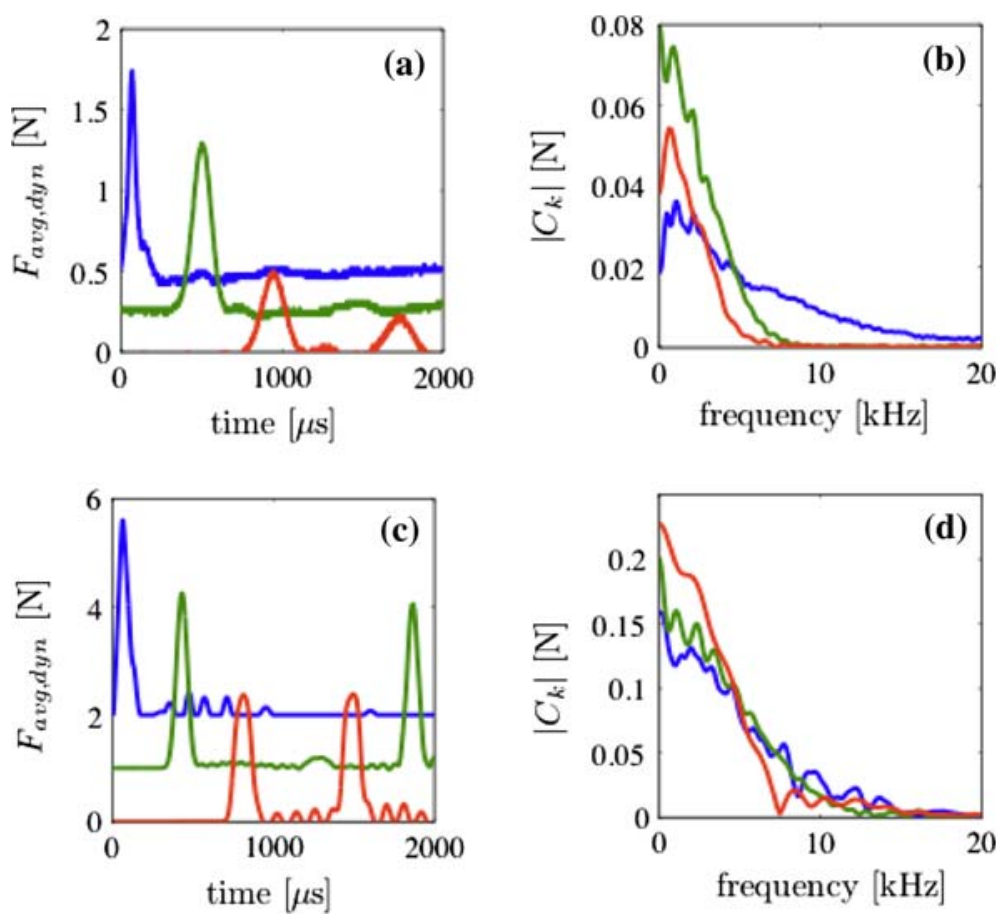

Fig. 11 Strongly nonlinear case with only a gravitational static load. The striker had a mass equal to one cell $(0.61 \mathrm{~g})$ and an initial velocity of $0.44 \mathrm{~m} / \mathrm{s}$. Experimental $(\mathbf{a}, \mathbf{b})$ and numerical $(\mathbf{c}, \mathbf{d})$ data on the propagation of strongly nonlinear solitary waves in a diatomic chain composed of 37 elements. Curves show the force versus time behavior detected by the sensors in the 2 nd particle (top curve), 14th particle (second curve from top), 27th particle and at the wall (bottom curve). The time begins at the moment of impact and is triggered by the first pulse on the oscilloscope
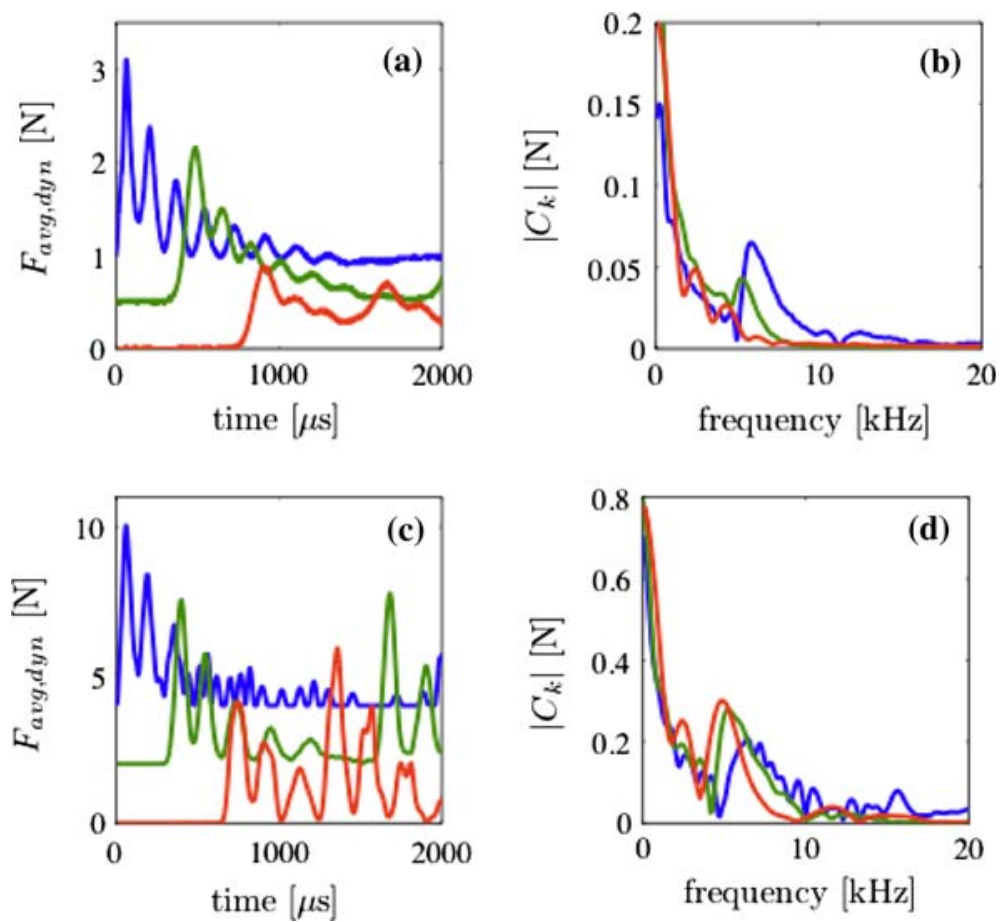

Fig. 12 Strongly nonlinear case, there is no initial compression and only gravity is included. The striker had a mass equal to 4.5 cells $(2.75 \mathrm{~g})$ and an initial velocity of $0.44 \mathrm{~m} / \mathrm{s}$. Experimental $(\mathbf{a}, \mathbf{b})$ and numerical $(\mathbf{c}, \mathbf{d})$ data on the propagation of strongly nonlinear solitary waves in a diatomic chain composed of 37 elements: the curves show the force vs. time behavior detected by the sensors in the 2nd particle (top curve), 14th particle (second from top), 27th particle (third from top) and at the wall (bottom curve) 

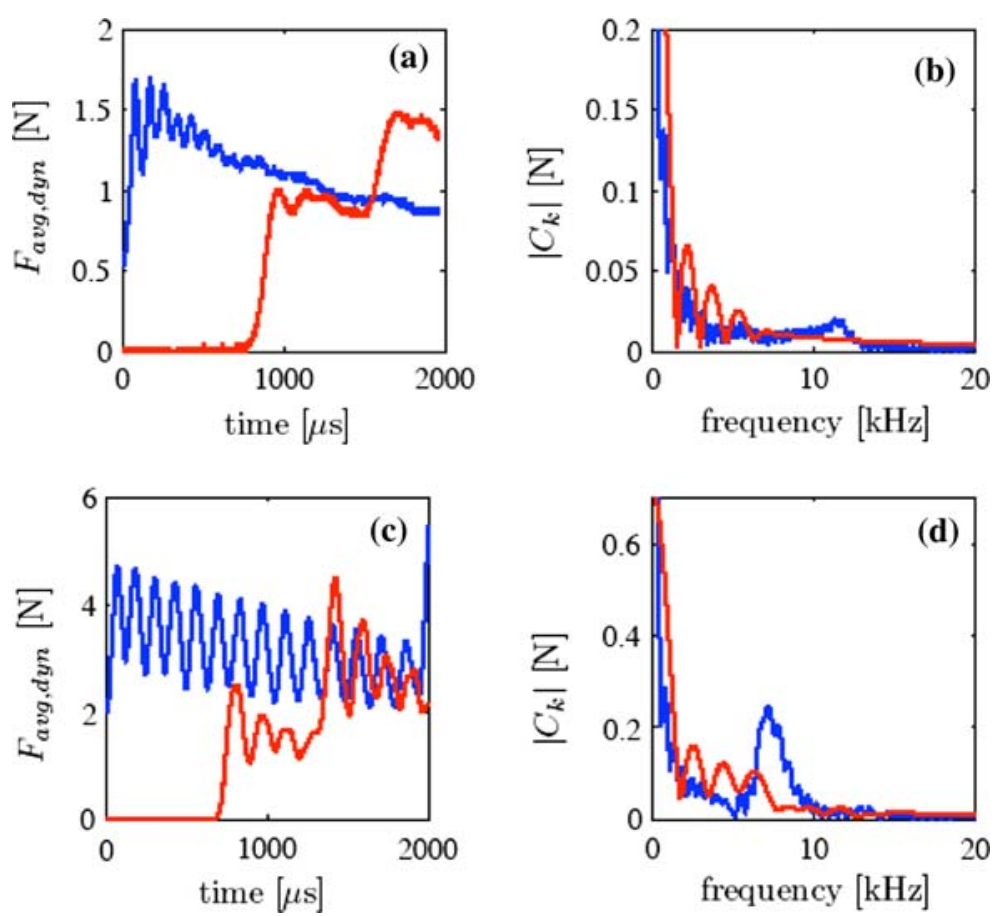

Fig. 13 Oscillatory shock-like waves in strongly nonlinear case; there is no initial compression and only gravity is included. The striker had a mass equal to $17.81 \mathrm{~g}$ and an initial velocity of $0.2 \mathrm{~m} / \mathrm{s}$. Experimental (a, b) and numerical (c, d) data on the propagation of strongly nonlinear solitary waves in a diatomic chain composed of 37 elements (no precompression other than the gravitational preload is included). The Curves show the force versus time behavior detected by the sensors in the 2nd particle (top curve), 27th particle (second from the top) and at the wall (bottom curve). b Corresponding frequency plots. c Numerical calculation corresponding to $\mathbf{a}, \mathbf{d}$ frequency spectrum obtained in the numerical calculations

well with the solitary wave width predicted in the long wave approximation for a chain of particles interacting according to Hertz's law, which predicts a wavelength of approximately five cell lengths [31,37]. It is similar to the behavior of a chain with equal masses where the number of solitary waves is determined by the ratio of impactor mass to the mass of the particle $[15,31,38]$. This result corresponds well with numerical results presented in Fig. 11c and Table 2. There are several smaller pulses trailing the main pulse apparent in the top curve of Fig. 11a and c. These small pulses result from multiple impacts of the first PTFE particle from the top magnetic particle and first stainless steel cylinder.

The Fourier spectrum for the strongly solitary wave and the signal in the nonlinear system (compare Fig. 8b, d with Fig. 11b, d) are comparable due to the similar durations of the initial pulses. It should be mentioned that the speed of the linear, weakly nonlinear and strongly nonlinear signals in these cases are different but of the same order of magnitude.

The frequency spectrum shifts toward lower frequencies as the pulse propagates into the chain. This is due to the formation of the solitary wave being a stationary solution [43] of the long-wave approximation with a spectrum of frequencies being close to the band-gap range presented in the previous section for the precompressed chain. This coincidence is due to the specific value of the striker velocity creating an amplitude of pulses comparable to the initial precompression in previous cases which results in similar sound speed and solitary wave speeds. A more pronounced shift toward lower frequencies in experiments (Fig. 11b) with a single pulse is attributed to amplitude attenuation resulting in longer pulse duration with the same spatial width.

A similar behavior of signals in the frequency domain both in experiments and numerical calculations was observed for pulses excited by the impact of an alumina striker with larger mass $(1.22 \mathrm{~g}$ at $0.44 \mathrm{~m} / \mathrm{s})$ generating a series of pulses.

In Fig. 12 the experimental and numerical results of the impact of an alumina striker $(2.75 \mathrm{~g}$ at $0.44 \mathrm{~m} / \mathrm{s})$ are presented. This impact created about 8 pulses in experiments, and numerical calculations and the corresponding frequency spectrum of the propagating pulses are shown in Fig. 12b and d. At the 27th (stainless steel cylinder) particle it is clear that the spectrum has shifted to lower frequencies. 
In Fig. 13 the experimental and numerical results of the impact of an alumina striker $(17.81 \mathrm{~g}$ at $0.2 \mathrm{~m} / \mathrm{s})$ are presented. This impact created oscillatory shock-like waves in experiments and numerical calculations, and the corresponding frequency spectrums of the incoming pulses are shown in Fig. 13b and d. The incoming oscillatory shock-like wave becomes almost monotonic by the 27th (PTFE) particle in experiments, and a similar trend is evident in numerical calculations, which was also observed in case of the nonlinear chain (compare Figs. 10a, c and 13a, c), despite the fact that dissipation was not included in the calculations. The mechanism of changing the oscillatory character of the shock wave without dissipation may be similar to that discussed earlier for the case of a nonlinear chain. The observed transformation of the frequency spectrum is similar to the nonlinear case and may be due to the effects of an in situ band-gap induced by the dynamic compression behind a relatively long shock-like wave resulting in fast attenuation of harmonics with frequencies inside the band gap which were initially excited by the impact. In a one mass chain such transformation of an oscillatory shock-like wave in absence of dissipation was not observed [49].

The qualitative influence of dissipation was investigated in this case assuming that the Coulomb friction of Teflon balls on a Teflon wall supporting them does not introduce significant losses in comparison with losses on the contact areas because forces between Teflon balls and the Teflon wall in the direction perpendicular to the chain are very small and the coefficient of friction is very low. Also Coulomb type of dissipation is neglected since the displacement of the individual elements in the chain is small. This is supported by experimental observations in our other experiments with steel balls in the same Teflon tube where friction is expected to be more pronounced, but we did not observe this level of attenuation as in the chain of PTFE balls [33]. For this reasons we connect the dissipation phenomenon to the contact processes in the chain of viscoelastic PTFE balls according to the mechanism proposed in [47] and not with the Coulomb friction between the wall and balls. To demonstrate the qualitative effect of nonlinear dissipation on the shape of the signal we used the corresponding equations for a chain with nonlinear dissipation presented below:

$$
\begin{aligned}
m_{n} \ddot{u}_{n}= & A\left(u_{n-1}-u_{n}\right)^{3 / 2}-A\left(u_{n}-u_{n+1}\right)^{3 / 2} \\
& +3 B / 2\left(u_{n-1}-u_{n}\right)^{1 / 2}\left(\dot{u}_{n-1}-\dot{u}_{n}\right) \\
& -3 B / 2\left(u_{n}-u_{n+1}\right)^{1 / 2}\left(\dot{u}_{n}-\dot{u}_{n+1}\right) .
\end{aligned}
$$

In [47] the coefficient $B$ is related to viscoelastic properties of the material of the ball which are unknown for PTFE for the conditions of our experiments; masses of particles are different depending on index $n$. For this reasons we used the coefficient $B=3 \times 10^{-10}$ which gave the transformation of the signal qualitatively similar to observations in experiments. More detailed research of the dissipation mechanism is necessary to get better quantitative agreement between the amplitudes and the shape of the signal in calculations and experiments.

\section{Conclusions}

One-dimensional diatomic nonlinear chains have been assembled and tested using PTFE spheres and steel cylinders. The band-gap frequencies can be achieved inside the audible range of frequencies and adjusted by the mass of the particles, their elastic modulus and magnetically induced initial static compression. The transformation of pulses with frequencies inside the phononic band-gap (evanescent waves) is observed in numerical calculations in statically compressed chains. It was shown that the band-gap rapidly transforms incoming pulses within very short distances from the source in a linear and nonlinear elastic chain. We explained that the band gap effect has significant influence on signal transformation because (a) the cutoff frequency (the lower bound) is observed at roughly the same location when comparing the numerical calculations and experiments and (b) because of the order of magnitude difference in the characteristic distances for evanescent and dissipation effects. As a result we have observed that the cutoff frequency effects were developed very quickly, faster than effects due to dissipation.

It was demonstrated that the band-gap calculated in the linear approximation is relevant also to transformation of quasiharmonic signals with large amplitude corresponding to nonlinear behavior of the system. Initial disturbances separated into two groups of waves; pure acoustic and trailing group of signals with optical frequencies.

Strongly nonlinear solitary waves with a width of five cells were observed in experimental and numerical calculations as predicted by the long wave approximation. The experimental results demonstrate a qualitative change of the propagating wave properties under different conditions of static compression. With the magnetically induced compression, the speed of the solitary wave was increased by $50 \%$. 
Oscillatory shock-like waves were excited under the impact of the striker in nonlinear and strongly nonlinear systems. They demonstrated a transition to reduced frequency oscillations both in experiments and calculations without dissipation. This may be explained by the in situ band-gap generated by dynamic compression behind a relatively long shock wave. The increased frequency of harmonics in incoming shock profiles in experiments in comparison with numerical calculations may be due to dissipation.

Acknowledgment This work was supported by NSF (Grant No. DCMS03013220).

Open Access This article is distributed under the terms of the Creative Commons Attribution Noncommercial License which permits any noncommercial use, distribution, and reproduction in any medium, provided the original author(s) and source are credited.

\section{References}

1. Tasi, J.: Initial-value problems for nonlinear diatomic chains. Phys. Rev. B 14, 2358 (1976)

2. Pnevmatikos, St., Remoissenet, M., Flytzanis, N.: Propagation of acoustic and optical solitons in nonlinear diatomic chains. J. Phys. C: Solid State Phys. 16, L305 (1983)

3. Pnevmatikos, St., Flytzanis, N., Remoissenet, M.: Soliton dynamics of nonlinear diatomic lattices. Phys. Rev. B 33, 2308 (1986)

4. Chubykalo, O.A., Kovalev, A.S., Usatenko, O.V.: Dynamical solitons in a one-dimensional nonlinear diatomic chain. Phys. Rev. B 47, 3153 (1993)

5. Tchawoua, C., Kofane, T.C., Bokosah, A.S.: Dynamics of solitary waves in diatomic chains with long-range Kac-Baker interactions. J. Phys. A Math. Gen. 26, 6477 (1993)

6. Parmley, S., Zobrist, T., Clough, T., Perez-Miller, A., Makela, M., Yu, R.: Phononic band structure in a mass chain. Appl. Phys. Lett. 67, 777 (1995)

7. Franchini, A., Bortolani, V., Wallis, R.F.: Intrinsic localized modes in the bulk and at the surface of anharmonic diatomic chains. Phys. Rev. B 53, 5420 (1996)

8. Gorbach, A.V., Johansson, M.: Discrete gap breathers in a diatomic Klein-Gordon chain: Stability and mobility. Phys. Rev. E 67, 066608 (2003)

9. Maniadis, P., Zolotaryuk, A.V., Tsironis, G.P.: Existence and stability of discrete gap breathers in a diatomic $\beta$ Fermi-PastaUlam chain. Phys. Rev. E 67, 046612 (2003)

10. Lüerßen, D., Easwar, N., Malhotra, A., Hutchins, L., Schulze, K., Wilcox, B.: A demonstration of phonons that implements the linear theory. Am. J. Phys. 72, 197 (2004)

11. Kittel, C.: Introduction to Solid State Physics, Chap. 4. Wiley, New York (2005)

12. Hladky-Hennion, A.C., Allan, G., de Billy, M.: Localized modes in a one- dimensional diatomic chain of coupled spheres. J. Appl. Phys. 98, 054909 (2005)

13. Shen, M., Cao, W.: Acoustic band-gap engineering using finite-size layered structures of multiple periodicity. Appl. Phys. Lett. 75, 3713 (1999)

14. Alastalo, A.T., Kiihamaki, J., Seppa, H.: Microelectromechanical delay lines with slow signal propagation. J. Micromech. Microeng. 16, 1854 (2006)

15. Nesterenko, V.F.: Propagation of nonlinear compression pulses in granular media. Prikl. Mekh. Tekh. Fiz. 24, 136 (1983); J. Appl. Mech. Tech. Phys. 24, 733 (1984)

16. Lazaridi, A.N., Nesterenko, V.F.: Observation of a new type of solitary waves in a one-dimensional granular medium. Prikl. Mekh. Tekh. Fiz. 26, 115 (1985); J. Appl. Mech. Tech. Phys. 26, 405 (1985)

17. Nesterenko, V.F.: Nonlinear waves in "sonic vacuum". Fizika gorenia i vzryva (in Russian) 28, 121 (1992)

18. Shukla, A., Sadd, M.H., Xu, Y., Tai, Q.M.: Influence of loading pulse duration on dynamic load transfer in a simulated granular medium. J. Mech. Phys. Solids 41, 1795 (1993)

19. Nesterenko, V.F.: Solitary waves in discrete media with anomalous compressibility and similar to "sonic vacuum". J. Phys. IV Colloq 4, C8-729 (1994)

20. Friesecke, G., Wattis, J.A.D.: Existence theorem for solitary waves on lattices. Commun. Math. Phys. 161, 391 (1994)

21. Nesterenko, V.F., Lazaridi, A.N., Sibiryakov, E.B.: The decay of soliton at the contact of two "acoustic vacuums". Prikl. Mekh. Tekh. Fiz. 36, 19 (1995); J. Appl. Mech. Tech. Phys. 36, 166 (1995)

22. Coste, C., Falcon, E., Fauve, S.: Solitary waves in a chain of beads under Hertz contact. Phys. Rev. E 56, 6104 (1997)

23. Sen, S., Manciu, M., Wright, J.D.: Solitonlike pulse in perturbed and driven Hertzian chains and their possible applications in detecting buried impurities. Phys. Rev. E 57, 2386 (1998)

24. Chatterjee, A.: Asymptotic solution for solitary waves in a chain of elastic spheres. Phys. Rev. E 59, 5912 (1999)

25. Hong, J., Ji, J.-Y., Kim, H.: Power laws in non-linear granular chain under gravity. Phys. Rev. Lett. 82, 3058 (1999)

26. Hinch, E.J., Saint-Jean, S.: The fragmentation of a line of balls by an impact. Proc. R. Soc. Lond. A 455, 3201 (1999)

27. Coste, C., Gilles, B.: On the validity of Hertz contact law for granular material acoustics. Eur. Phys. J. B 7, 155 (1999)

28. Sen, S., Manciu, M.: Discrete Hertzian chains and solitons. Physica A 268, 644 (1999)

29. Manciu, M., Sen, S., Hurd, A.J.: The propagation and backscattering of soliton-like pulses in a chain of quartz beads and related problems. (I). Propagation. Physica A 274, 588 (1999)

30. Manciu, M., Sen, S., Hurd, A.J.: The propagation and backscattering of soliton-like pulses in a chain of quartz beads and related problems. (II). Backscattering. Physica A 274, 607 (1999)

31. Nesterenko, V.F.: Dynamics of Heterogeneous Materials, Chap. 1. Springer, New York (2001) 
32. Manciu, M., Sen, S., Hurd, A.J.: Impulse propagation in dissipative and disordered chains with power-law repulsive potentials. Physica D 157, 226 (2001)

33. Daraio, C., Nesterenko, V.F., Herbold, E., Jin, S.: Strongly nonlinear waves in a chain of Teflon beads. Phys. Rev. E 72, 016603 (2005)

34. Job, S., Melo, F., Sokolow, A., Sen, S.: How Hertzian solitary waves interact with boundaries in a 1-d granular medium. Phys. Rev. Lett. 94, 178002 (2005)

35. Nesterenko, V.F., Daraio, C., Herbold, E.B., Jin, S.: Anomalous wave reflection at the interface of two strongly nonlinear granular media. Phys. Rev. Lett. 95, 158702 (2005)

36. Hong, J.: Universal power-law decay of the impulse energy in granular protectors. Phys. Rev. Lett. 94, 108001 (2005)

37. Daraio, C., Nesterenko, V.F., Herbold, E., Jin, S.: Tunability of solitary wave properties in one-dimensional strongly nonlinear phononic crystals. Phys. Rev. E 73, 026610 (2006)

38. Sokolow, A., Bittle, E.G., Sen, S.: Solitary wave train formation in Hertzian chains. Europhys. Lett. 77, 24002 (2007)

39. Sen, S., Hong, J., Bang, J., Avalos, E., Doney, R.: Solitary waves in the granular chain. Phys. Rep. 462, 21 (2008)

40. Spence, D.A.: Self similar solutions to adhesive contact problems with incremental loading. Proc. R. Soc. London A, A 305, 55 (1968)

41. Goddard, J.D.: Nonlinear elasticity and pressure-dependent wave speeds in granular media. Proc. R. Soc. Lond. A 430, 105 (1990)

42. Storakers, B. Elaguine D.J.: Hertz contact at finite friction and arbitrary profiles. J. Mech. Phys. Solids 53, 1422 (2005)

43. Porter, M.A., Daraio, C., Herbold, E.B., Szelengowicz, I., Kevrekidis, P.G.: Highly nonlinear solitary waves in periodic dimer granular chains. Phys. Rev. E 77, 015601(R) (2008)

44. Porter, M.A., Daraio, C., Szelengowicz, I., Herbold, E.B., Kevrekidis, P.G.: Highly nonlinear solitary waves in heterogeneous periodic granular media. Physica D 238 (2009)

45. Tournat, V., Gusev, V.E., Castagnede, B.: Self-demodulation of elastic waves in a one-dimensional granular chain. Phys. Rev. E 70, 056603 (2004)

46. Coste, C., Gilles, B.: Sound propagation in a constrained lattice of beads: High-frequency behavior and dispersion relation. Phys. Rev. E 77, 021302 (2008)

47. Hertzsch, J.-M., Spahn, F., Brilliantov, N.V.: On low-velocity collisions of viscoelastic particles. J. Phys. II Fr. 5, 1725 (1995)

48. Carretero-Gonzalez, R., Khatri, D., Porter, M.A., Kevrekidis, P.G., Daraio, C.: Dissipative solitary waves in granular crystals. Phys. Rev. Lett. 102, 024102 (2009)

49. Herbold, E.B., Nesterenko, V.F.: Shock wave structure in a strongly nonlinear lattice with viscous dissipation. Phys. Rev. E 75, 021304 (2007)

50. Rosas, A., Romero, A.H., Nesterenko, V.F., Lindenberg, K.: Observation of two-wave structure in strongly nonlinear dissipative granular chains. Phys. Rev. Lett. 98, 164301 (2007)

51. Rosas, A., Romero, A.H., Nesterenko, V.F., Lindenberg, K.: Short-pulse dynamics in strongly nonlinear dissipative granular chains. Phys. Rev. E 78, 051303 (2008)

52. Duvall, G.E., Manvi, R., Lowell, S.C.: Steady shock profile in a one-dimensional lattice. J. Appl. Phys. 40, 3771 (1969)

53. Nahmad-Molinari, Y., Arancibia-Bulnes, C.A., Ruiz-Suarez, J.C.: Sound in a magnetorheological slurry. Phys. Rev. Lett. 82, 727 (1999) 